\title{
Clues about the Nature of Multiquark States
}

\author{
Humberto Garcilazo ${ }^{1,+}$ and Alfredo Valcarce ${ }^{2, *,+} \mathbb{D}$ \\ 1 Escuela Superior de Física y Matemáticas, Instituto Politécnico Nacional, Edificio 9, Mexico D.F. \\ 07738, Mexico; humberto@esfm.ipn.mx \\ 2 Departamento de Física Fundamental, Universidad de Salamanca, E-37008 Salamanca, Spain \\ * Correspondence: valcarce@usal.es \\ + These authors contributed equally to this work.
}

check for updates

Citation: Garcilazo, H.; Valcarce, A. Clues about the Nature of Multiquark States. Symmetry 2021, 13, 1171.

https://doi.org/10.3390/sym13071171

Academic Editor: Ignatios Antoniadis

Received: 7 June 2021

Accepted: 25 June 2021

Published: 29 June 2021

Publisher's Note: MDPI stays neutral with regard to jurisdictional claims in published maps and institutional affiliations.

Copyright: (c) 2021 by the authors. Licensee MDPI, Basel, Switzerland. This article is an open access article distributed under the terms and conditions of the Creative Commons Attribution (CC BY) license (https:// creativecommons.org/licenses/by/ $4.0 /)$.

\begin{abstract}
We present a comprehensive study of the decay width of multiquark states containing different color singlet components in a coupled-channel approach. We show how the decay width can provide in-depth information about the nature of a coupled-channel resonance. An unexpected behavior of the decay width of a multiquark state could be pointing to a relevant role of the coupledchannel dynamics, aiming at the channel responsible for the formation of the resonance. The symmetrical situation between meson- and baryon-like multiquarks is highlighted. Our study accounts for the existence of narrow resonances with large phase spaces. In the case of resonances far from their detection channel, it is the mass difference with the formation channel that determines their decay width. The larger the binding, the larger the decay width, even though the phase space to the detection channel gets reduced. The trends noticed cast doubts on the molecular assignment of some multiquark candidates. Finally, we wonder about the existence and properties of multiquark partners in other flavor sectors.
\end{abstract}

Keywords: multiquarks; quark models; few-body systems

\section{Rationale}

One of the most noteworthy aspects of current hadronic physics is the significant number of suggested experimental resonances whose nature could be closely related to multiquark states. It might be that hadronic spectroscopy presents a smooth transition from quark to hadron degrees of freedom where multiquarks are common structures. Multiquark candidates have been found in the meson, baryon number $B=0$, and baryon $B=1$, sectors with symmetric phenomenological findings. To understand the existence and properties of such potential multiquark states is an enormous challenge that would help to advance knowledge of Quantum Chromodynamics (QCD) in the low-energy nonperturbative regime. It is a common feature of most of the suggested multiquark states that, while they are experimentally detected in a particular lower energy channel, they are, however, also theoretically predicted as being bound states of a higher energy channel very close to their masses [1].

Overall, there is no clear-cut relation between the decay width of these resonances with the phase space available for the decay into their detection channel. One of the more notable instances arises in the case of the $\mathrm{LHCb}$ pentaquarks [2,3], whose properties are summarized in Table 1. Their $J / \psi p$ decays suggest that, regardless of the internal dynamics, the states have intrinsic quark content $c \bar{c} u d d$. As can be seen in Table 1, it is not easy to guess a relationship between the decay width and the available phase space into their common detection channel, $J / \psi p$. Note, for instance, that the resonance with the largest phase space has the smallest decay width and that resonances with similar phase spaces have widths that differ in orders of magnitude. Such behavior should draw our attention and potentially our concern. Symmetric situations also arise in the meson sector. See Ref. [1] and references therein for a recent review. 
Table 1. Summary of the $P_{c}^{+}$properties decaying to $J / \psi p[2,3]$.

\begin{tabular}{cccc}
\hline State & $\boldsymbol{M} \mathbf{( M e V )}$ & $\boldsymbol{\Gamma} \mathbf{( M e V )}$ & Phase Space $(\mathbf{M e V})$ \\
\hline$P_{c}(4380)^{+}$ & $4380 \pm 8 \pm 29$ & $205 \pm 18 \pm 86$ & $345 \pm 30$ \\
$P_{c}(4312)^{+}$ & $4311.9 \pm 0.7_{-0.6}^{+6.8}$ & $9.8 \pm 2.7_{-4.5}^{+3.7}$ & $276.7 \pm 6.8$ \\
$P_{c}(4440)^{+}$ & $4440.3 \pm 1.3_{-4.7}^{+4.1}$ & $20.6 \pm 4.9_{-10.1}^{+8.7}$ & $405.1 \pm 4.9$ \\
$P_{c}(4457)^{+}$ & $4457.3 \pm 0.6_{-1.7}^{+4.1}$ & $6.4 \pm 2.0_{-1.9}^{+5.7}$ & $422.1 \pm 4.1$ \\
\hline
\end{tabular}

In general, these resonances are scrutinized through models and/or arguments devised to reproduce their masses under particular hypotheses for their structure [1]. There is, however, no definitive conclusion about the nature of such states. Therefore, a coherent analysis of all available experimental data, including their decay width, could provide insights into their internal structure. This may also make clearer the behavior of the basic theory governing the quark dynamics-QCD—in the nonperturbative regime. One should bear in mind the success of nonrelativistic realizations of QCD, dominated by chromomagnetic and chromoelectric effects, for the description of the low-energy baryon and meson spectra. See, for example, Ref. [4] and references therein. This suggests the possibility of also trying to accommodate multiquark states in a similar picture that, as we will see, becomes more complicated due to the presence of open thresholds [5]. This makes the theoretical description more complicated and may be the right way forward for understanding the experimental data.

In this work, we perform a detailed study on the correlation between the mass and the decay width of a resonance in a coupled-channel approach. Our study is generic and therefore applicable to very different scenarios. We make use of an intuitive model that allows us to simulate different experimental situations, which will be discussed immediately below. Particular quantitative results would require a dedicated fit that is beyond the purpose of the present work but that could be easily envisaged within any available model.

\section{Experimental Scenario and Theoretical Framework}

\subsection{Experimental Scenario}

A large amount of the experimental data linked to multiquarks have been reported through decays to charmonium and bottomonium states, both in the $B=0$ and $B=1$ sectors. For $B=0$, they might correspond to a $Q \bar{Q} q \bar{q}$ quark-model structure, with $Q=c$ or $b$ and $q=u$ or $d$. Symmetrically, for $B=1$ the hypothetical multiquark structures would have a minimal quark content $Q \bar{Q} q q q$. The first system is made of two different types of color singlets: $[Q \bar{Q}][q \bar{q}]$ and $[Q \bar{q}][q \bar{Q}]$; analogously, the second system is also made of two different types of color singlets: $[Q \bar{Q}][q q q]$ and $[q \bar{Q}][Q q q]$. It is worth noting that there are already experimental candidates for $Q \bar{Q}^{\prime} q \bar{q}$ multiquark structures, where $Q=c$ and $Q^{\prime}=s[6]$. Because they also contain two different types of color singlet, our reasoning remains equally valid.

If the quark dynamics within a multiquark is not constrained due to the existence of highly correlated substructures, as it could be, for example, in the case of diquarks [7], the two color singlets will contribute to a multiquark meson,

$$
\Psi_{4 q}=\alpha_{1}|[Q \bar{Q}][q \bar{q}]\rangle+\alpha_{2}|[Q \bar{q}][q \bar{Q}]\rangle,
$$

where, in general, $\alpha_{1}$ and $\alpha_{2}$, determined by the quark dynamics, would be different from zero. A similar expression holds for multiquark baryons,

$$
\Psi_{5 q}=\beta_{1}|[Q \bar{Q}][q q q]\rangle+\beta_{2}|[q \bar{Q}][Q q q]\rangle .
$$

The above equations are written in non-orthogonal bases in color space, made of internal color singlets. They can be transformed to orthogonal bases, see Refs. [8,9] for details. Such a transformation is suited to dealing with the Pauli principle or for the 
evaluation of specific matrix elements. Each of the two components in Equation (1) and (2) can indeed evolve into the other one. One is dealing with a compact object whose internal color quantum numbers are not separately conserved during time evolution. In other words, these states are not generally simple bound states of two hadrons. Therefore, their properties can be addressed by means of a coupled-channel calculation involving the two different components of Equations (1) and (2). Beforehand, there is no dynamical reason that could justify the relevance of any of them relying on the overall principles of QCD. At this point, it is relevant to analyze the mass of the different color singlet components to illustrate how the coupled-channel dynamics would be affected depending on the flavor sector one is working with.

As mentioned above, multiquarks with a $Q \bar{Q} q \bar{q}$ flavor content contain $[Q \bar{q}][q \bar{Q}]$ and $[Q \bar{Q}][q \bar{q}]$ color singlets that are coupled together through the internal quark dynamics. For $Q=c$, the $[Q \bar{Q}][q \bar{q}]$ and $[Q \bar{q}][q \bar{Q}]$, color singlets have masses close to each other, while for $Q=b$ the $[Q \bar{Q}][q \bar{q}]$ mass is much lower than that in the charm sector, due to a larger binding energy. It is also much lower than the $[Q \bar{q}][q \bar{Q}]$ one (see Figure 1 ). The growth of the mass difference between the two color singlet components in Equation (1), when increasing the mass of the heavy quark, is linked to the flavor-independence of the chromoelectric interaction $[5,10]$, because the binding energy is proportional to the reduced mass of the interacting pair. Thus, for large $M_{Q}$, while the binding of the $[Q \bar{q}][q \bar{Q}]$ color singlet would be roughly proportional to $2 m_{q}$, that of the $[Q \bar{Q}][q \bar{q}]$ color singlet is proportional to $\left(M_{Q}+m_{q}\right) / 2$, the mass difference increasing linearly with the mass of the heavy quark. As can be seen in Figure 1, in the strange sector the reverse is true. This is because the mass of the strange quark is smaller and has a more involved quark dynamics. As a consequence, in the strange sector the $[Q \bar{q}][q \bar{Q}]$ component becomes the lowest in energy, thereby opening the way to stable meson-antimeson molecules [11]. For the charm and bottom heavy flavor sectors, these bound states would correspond to resonances in between the two internal color singlet components.

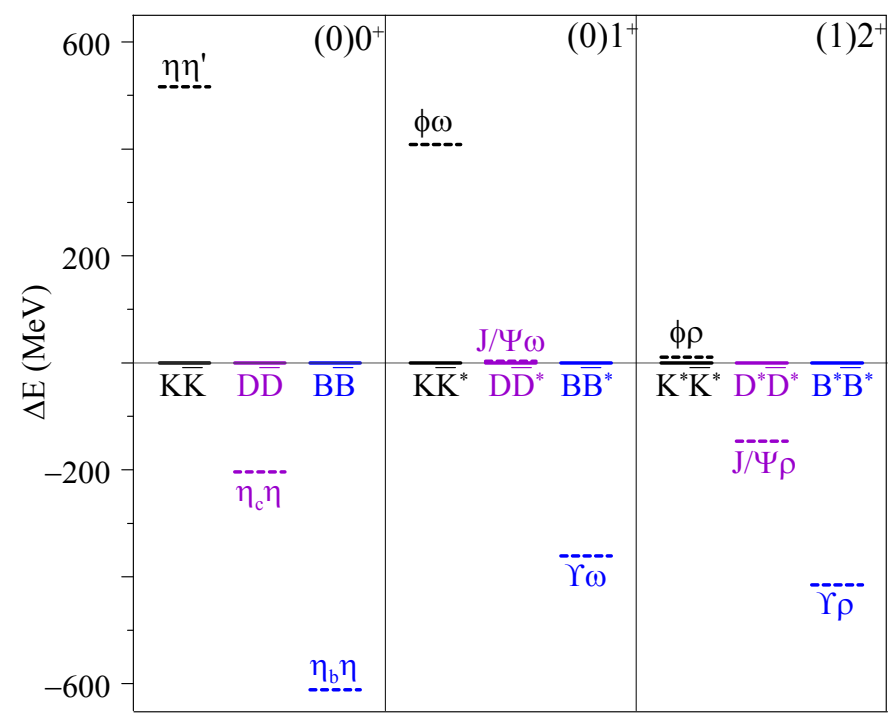

Figure 1. Experimental masses [12] of the different color singlets that make up $Q \bar{Q} q \bar{q}$ multiquarks with $Q=s, c$, or $b$, for different sets of quantum numbers, $(I) J^{P}$. We display several cases containing pseudoscalar-pseudoscalar, pseudoscalar-vector and vector-vector $[Q \bar{q}][q \bar{Q}]$ meson-antimeson components. The reference energy has been set to the corresponding $K \bar{K}, D \bar{D}$ or $B \bar{B}$ mass for the hidden strange, charm and bottom sectors, respectively.

The flavor independence of the chromoelectric interaction produces a symmetric effect for $Q \bar{Q} q q q$ multiquarks. The binding of the $[Q \bar{Q}][q q q]$ color singlet is controlled by $M_{Q}$ while that of the $[q \bar{Q}][Q q q]$ color singlet is regulated by $m_{q}$. Hence, in the charm sector for $J=1 / 2$, the mass difference $\Delta M=M_{[q \bar{Q}][Q q q]}-M_{[Q \bar{Q}][q q q]}=M_{\bar{D} \Sigma_{c}}-M_{J / \psi p}=288 \mathrm{MeV}$; howeve, 
$\mathrm{r}$ in the bottom sector it increases to $\Delta M=M_{[q \bar{Q}][Q q q]}-M_{[Q \bar{Q}][q q q]}=M_{\bar{B} \Sigma_{b}}-M_{Y p}=692$ $\mathrm{MeV}$ [12]. This situation is illustrated in Figure 2 for $J^{P}=1 / 2^{+}$and $J^{P}=3 / 2^{+}$multiquark baryons of the charm and bottom sectors.

In a coupled-channel calculation, the non-diagonal terms are proportional to the matrix elements of the coupled channels and to the inverse of their mass difference. Assuming a dominant flavor-independent interaction, as would happen with the chromoelectric potential in the heavy-flavor sector, the larger the mass of the heavy flavor the weaker the effective coupling between the coupled channels. The most relevant consequence derived from Figures 1 and 2 is precisely that the mass difference between the coupled channels varies a lot depending on the flavor sector. This becomes particularly evident in the meson sector for the quantum numbers of the $X(3872)$, where the two color singlets are almost degenerate in the charm sector, while they are far apart in the bottom sector. Therefore, similar structures of different flavor sectors may give rise to very different outcomes. Hence, a certain caution is needed to predict partners of multiquarks in different flavor sectors if the dynamics are almost flavor independent and become governed by coupled-channel effects.

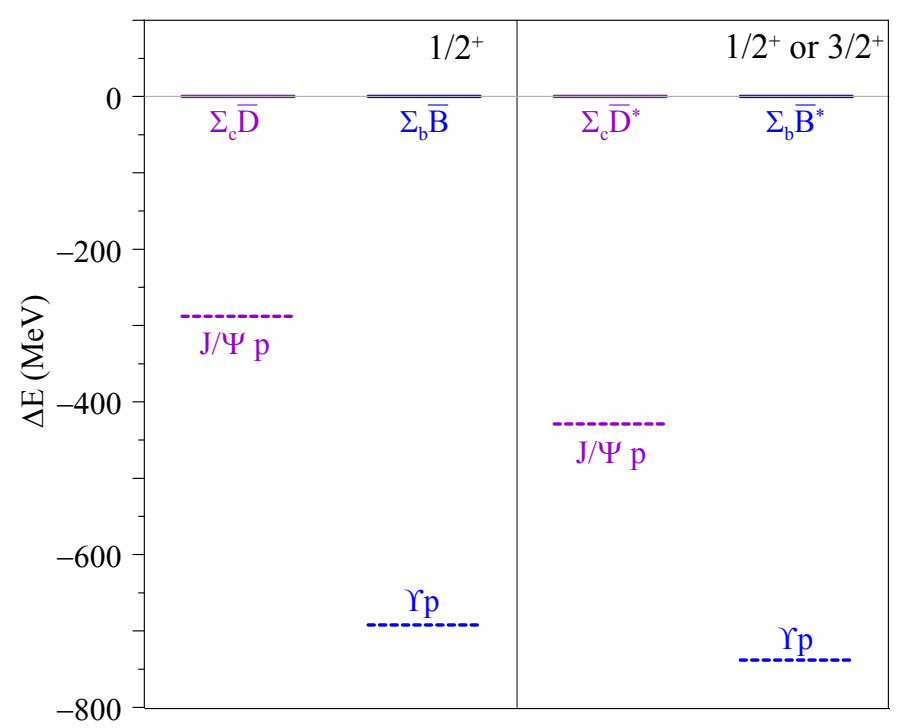

Figure 2. Experimental masses [12] of the different color singlets that make up selected $Q \bar{Q} q q q$ multiquarks with $Q=c$ or $b$, for different sets of quantum numbers, $J^{P}$. The reference energy has been set to the corresponding $\Sigma_{c} \bar{D}$ or $\Sigma_{b} \bar{B}$ mass for the hidden charm and bottom sectors, respectively.

The above reasoning requires an in-depth analysis of the dependence of the decay width of a resonance in terms of the color singlets that it is made of, and their internal coupling, in other words, the values of $\alpha_{1}$ and $\alpha_{2}$ in Equation (1), or $\beta_{1}$ and $\beta_{2}$ in Equation (2). As these equations can be formally written in a symmetric manner,

$$
\Psi_{n q}=\gamma_{1}\left|H_{1} H_{2}\right\rangle+\gamma_{2}\left|H_{3} H_{4}\right\rangle,
$$

in both cases we are facing a coupled-channel problem with two internal physical thresholds. Experimentally, most parts of the multiquark candidates appear in between the two color singlet components and this is thus the energy region where we will concentrate our analysis. Moreover, the quark dynamics suggests that the resonance is generated by the attraction in the channel with a larger mass, having a lower mass channel open. This situation is similar to a Feshbach resonance, where the open channel is represented by the $[Q \bar{Q}][q \bar{q}]$ or $[Q \bar{Q}][q q q]$ states that would get trapped in a molecular state supported by the closed channel potentials $[Q \bar{q}][q \bar{Q}]$ or $[q \bar{Q}][Q q q][13,14]$. 


\subsection{Theoretical Framework}

Let us then sketch the formalism we shall use to study the problem discussed in the previous section and represented diagrammatically in Figure 3. We are dealing with a coupled-channel problem of two different two-body color singlets with a resonance that is produced in between them. Channel 1, the lower in mass, is made of two color singlets, $[Q \bar{Q}][q \bar{q}]$ for $B=0([Q \bar{Q}][q q q]$ for $B=1)$. We denote by $m_{1}$ the mass of the $Q \bar{Q}$ state and by $m_{2}$ that of the $q \bar{q}(q q q)$ state. Channel 2, the higher in mass, consists of another two color singlets, $[Q \bar{q}][q \bar{Q}]$ for $B=0([q \bar{Q}][Q q q]$ for $B=1)$. We denote by $m_{3}$ the mass of the $Q \bar{q}$ state and by $m_{4}$ that of the $q \bar{Q}(Q q q)$ state. There is a mass difference $\Delta M$ between these two channels. As has been discussed above, the two color singlet components form a non-orthogonal basis in color space [8,9]. The interacting potentials between the different color singlets could be derived from a particular quark model, see for example [15], but they have to follow the general trends described in Section 2.1: an attractive upper channel that would have a bound state in a single-channel calculation, a weakly interacting lower channel that does not move the resonance below its mass, and a transition potential that can be regulated to make it softer when increasing the heavy-quark mass. Thus, the attractive character of the upper channel is shaped through the existence of a low-energy bound state, with a binding energy $\Delta E$. This bound state becomes a resonance for a lower open channel. The resonance would be detected by its decay to channel 1 , which implies that the upper and lower channels must be connected by some transition potential $V^{i j}$. Therefore, we have modeled the system as a coupled-channel problem obeying the non-relativistic Lippmann-Schwinger equation, which in the case of $S$-wave interactions is written as,

$$
\begin{aligned}
t^{i j}\left(p, p^{\prime} ; E\right) & =V^{i j}\left(p, p^{\prime}\right)+\sum_{k=1,2} \int_{0}^{\infty} p^{\prime \prime 2} d p^{\prime \prime} \\
& \times V^{i k}\left(p, p^{\prime \prime}\right) \frac{1}{E-\Delta M \delta_{2, k}-p^{\prime \prime 2} / 2 \mu_{k}+i \epsilon} t^{k j}\left(p^{\prime \prime}, p^{\prime} ; E\right), \quad i, j=1,2,
\end{aligned}
$$

where $\mu_{1}=m_{1} m_{2} /\left(m_{1}+m_{2}\right)$ and $\mu_{2}=m_{3} m_{4} /\left(m_{3}+m_{4}\right)$ are the reduced masses of channels 1 and 2 , and $\Delta M=m_{3}+m_{4}-m_{1}-m_{2}$ with $m_{3}+m_{4}>m_{1}+m_{2}$. The interactions in momentum space are given by,

$$
V^{i j}\left(p, p^{\prime}\right)=\frac{2}{\pi} \int_{0}^{\infty} r^{2} d r j_{0}(p r) V^{i j}(r) j_{0}\left(p^{\prime} r\right),
$$

where the two-body potentials, which are inputs to our present study, consist of an attractive and a repulsive Yukawa term, that is,

$$
V^{i j}(r)=-A^{i j} \frac{e^{-\mu_{A}^{i j} r}}{r}+B^{i j} \frac{e^{-\mu_{B}^{i j} r}}{r} .
$$

This type of parametrization is known to work rather well for the study of two-, three-, and few-baryon systems $[16,17]$ and it is adopted here. We have considered scenarios where a resonance exists at an energy $E=E_{R}$ such that the phase shift $\delta\left(E_{R}\right)=90^{\circ}$, for energies between the masses of channels 1 and 2, that is, $0<E_{R}<\Delta M$. The mass of the resonance would be given by $W_{R}=E_{R}+m_{1}+m_{2}$. The width of the resonance is calculated using the Breit-Wigner formula as [18-20],

$$
\Gamma(E)=\lim _{E \rightarrow E_{R}} \frac{2\left(E_{R}-E\right)}{\operatorname{cotg}[\delta(E)]} .
$$

Let us finally note that, although the Breit-Wigner formula is not very accurate close to threshold, we have explicitly checked-by way of analytic continuation of the T-matrix in the second Riemann sheet- that at low energy the width follows the expected $\Gamma \sim E^{1 / 2}$ behavior. Far from threshold, the values of the width obtained from the Breit-Wigner formula agree reasonably well with the values obtained from the position of the pole of the 
$T$-matrix in the complex plane $E=E_{0}-\frac{i}{2} \Gamma$. In order to find the position of the pole for a given value of the energy $E$ in Equation (4) one can use the standard contour rotation method $p \rightarrow p e^{-i \phi}$, which opens large sections of the second Riemann sheet. However, as one approaches the lower threshold, the real part of the pole $E_{0}$ becomes negative and the simple contour rotation method does not work so that one must devise more complicated contours in order to avoid the branch cut starting at $E=E_{0}-\frac{i}{2} \Gamma$.

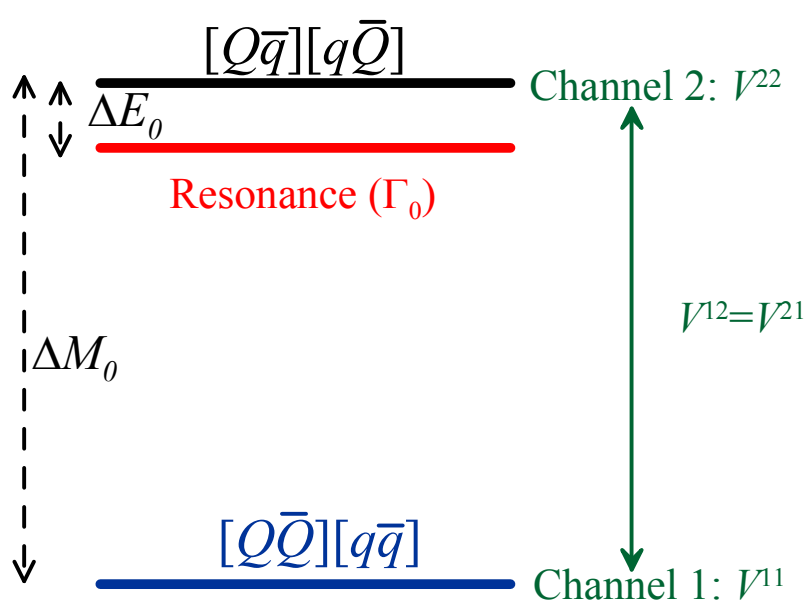

Figure 3. Diagrammatic representation of the mimicked experimental situation for $B=0$. A similar plot would apply to the $B=1$ sector replacing $[Q \bar{q}][q \bar{Q}]$ by $[q \bar{Q}][Q q q]$ and $[Q \bar{Q}][q \bar{q}]$ by $[Q \bar{Q}][q q q]$. See text for details.

\section{Results}

Without loss of generality, we make use of the same threshold masses recently considered in two- and three-body calculations [21,22]. None of the results that we will later discuss critically depend on the choice of the masses of the particles constituting the channels depicted in Figure 3. As said above, the interaction in the different channels is described by Yukawa potentials consisting of an attractive and a repulsive term. By varying the parameters, one is able to control the existence of a bound state or a resonance and its relative position with respect to the thresholds.

In order to fulfill the scenario depicted above that could have been addressed within a particular quark model [15], we start by requiring that, in a single-channel calculation, the upper channel 2 has a bound state with nearly zero energy, while in a coupled-channel calculation, the full system has a bound state only at the lower channel 1 . If one increases, for example, the magnitude of the repulsive term in the lower channel, $B^{11}$ in Table 2, the bound state of the coupled-channel system moves up and actually becomes a resonance in the continuum. In this way, one can study the behavior of the width of the resonance when its mass evolves from the lower channel 1 to the upper channel 2 in different situations. The starting point would be that shown in Figure 3-a resonance close to the upper channel-which corresponds to the parameters given in Table 2. Numerically, $\Delta E_{0}=3.64$ $\mathrm{MeV}, \Gamma_{0}=9.6 \mathrm{MeV}$ and $\Delta M_{0}=25.6 \mathrm{MeV}$.

Table 2. Parameters of the interaction as given in Equation (6). $A^{i j}$ and $B^{i j}$ are in $\mathrm{MeV}$ fm, while $\mu_{A}^{i j}$ and $\mu_{B}^{i j}$ are in $\mathrm{fm}^{-1}$.

\begin{tabular}{ccccc}
\hline$i j$ & $A^{i j}$ & $\boldsymbol{\mu}_{A}^{i j}$ & $B^{i j}$ & $\boldsymbol{\mu}_{B}^{i j}$ \\
\hline 11 & 100 & 2.68 & 3000 & 5.81 \\
22 & 680 & 4.56 & 642 & 6.73 \\
$12=21$ & 200 & 1.77 & 195 & 3.33 \\
\hline
\end{tabular}




\subsection{Dependence of the Decay Width on the Strength of the Transition Potential $V^{i j}$}

As has already been mentioned above, there is a noteworthy aspect of multiquark resonances. Whereas the dynamics seems to be dominated by the attraction in an upper channel where the resonance appears as a bound state, the tool for detection, however, is a lower channel where the strength of the interaction is rather weak if not negligible. In the case of pentaquarks, for instance, there is no known S-wave binding mechanism for charmonium-nucleon, $[Q \bar{Q}][q q q]$, combinations $[3,23]$ (the existence of an attractive potential in the lower channel might lead to bound states that would become deep in energy and therefore extremely narrow, due to the coupling to the upper channel. Neither the width nor the kinematical region would correspond to the experimental situation discussed in Section 2.1). Besides, the measured decay width of the resonances does not follow a $q^{2 \ell+1}$ behavior, typical for an elastic resonance where $q$ is the available momentum for the decay. Due to the necessary rearrangement of quarks inside the color singlets corresponding to arbitrary normalized values of $\alpha_{i}$ and $\beta_{i}$ in Equations (1) and (2), the coupling potential $V^{12}=V^{21}$ is short-ranged and weak. Then, our first quest is the evolution of the width of a coupled-channel resonance with a fixed position with respect to the formation and detection channels (channels 2 and 1 ) in terms of the strength of the coupling between them. For this purpose, we start from the initial conditions drawn at the beginning of this section for a given strength of the transition potential, $V_{0}^{12}$. We begin to lessen the strength of the transition potential but keep the resonance position fixed by either increasing the attraction in the upper channel or the repulsion in the lower one, obtaining a similar qualitative outcome. The results are shown in Figure 4.

Note that the phase space for the decay to the detection channel—channel 1-has been kept constant; however, the coupled channel dynamics change the behavior of the width compared to an elastic resonance, where it would remain constant. The trend obtained in Figure 4 would show up for any value of $\Delta E$. It is then clear that a coupled-channel resonance might be rather narrow despite a large phase space, merely because of a weak coupling between the formation and the detection channels.

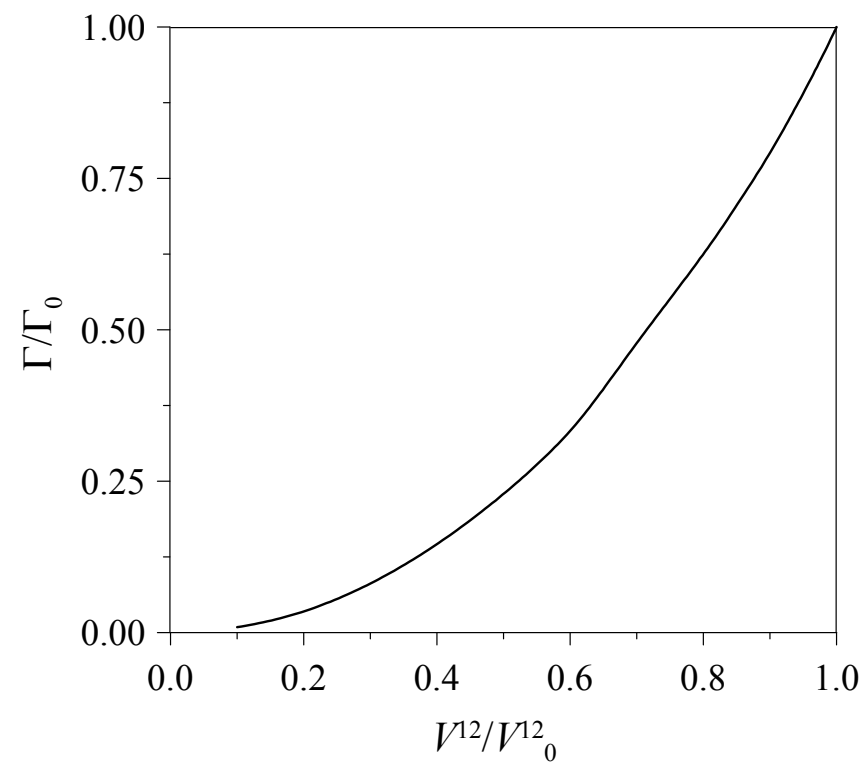

Figure 4. Variation of the decay width of a resonance, $\Gamma$ (in units of $\Gamma_{0}$ ), as a function of the strength of the coupling potential $V^{12}$ (in units of $V_{0}^{12}$ ) keeping $\Delta E_{0}$ fixed.

\subsection{Dependence of the Decay Width on $\Delta M_{0}-\Delta E$}

Let us now study the evolution of the decay width of the resonance as its position is varied in between the lower and upper channels. Put another way, the phase space to the decay channel and the binding energy to the formation channel are simultaneously modified. Because the resonance is generated by the attraction in the upper channel, we 
will only modify the repulsion in the detection channel to move its position. The results are depicted in Figure 5. The same trend is obtained for different strengths of $V^{12}$ or $V^{22}$. The width of the resonance starts increasing quickly when getting closer to the lower channel, although the phase space is reduced. However, about two thirds of the way towards the lower channel, the width starts to decrease. It is therefore worth noting that, for resonances that are far apart from their detection channel, it is the mass difference with respect to the formation channel which determines the width of the resonance: the larger the binding the larger the width, despite the reduction of the available phase space.

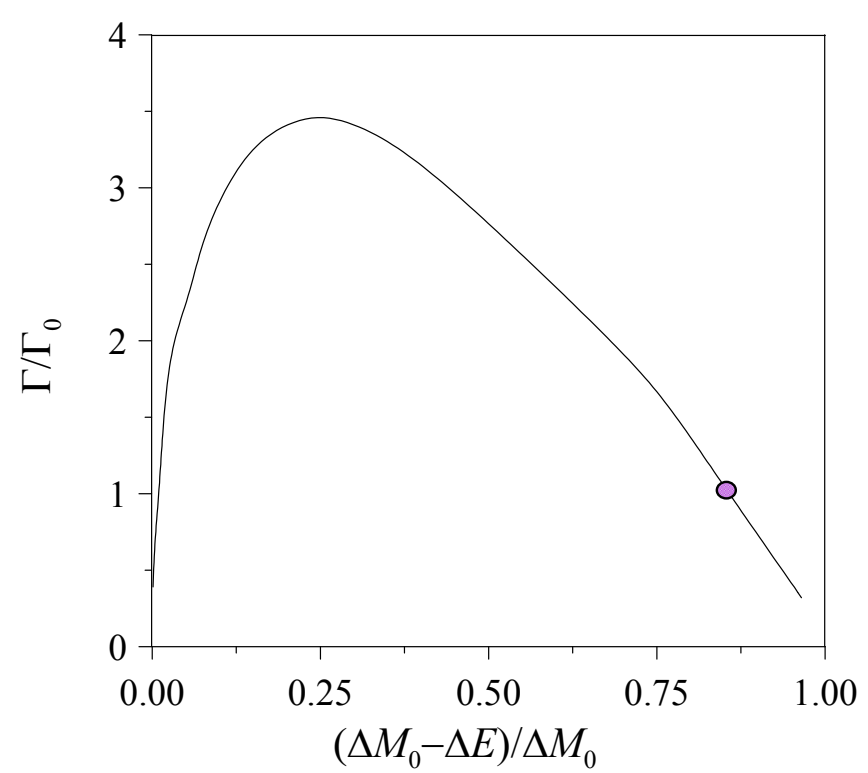

Figure 5. Variation of the decay width of a resonance, $\Gamma$ (in units of $\Gamma_{0}$ ), as a function of its relative position in between the formation and the detection channels, $\Delta M_{0}-\Delta E$ (in units of $\Delta M_{0}$ ). The purple circle indicates our starting point shown in Figure 3 and discussed at the beginning of Section 3.

When the resonance approaches the upper channel, it becomes narrow and seemingly ignores the existence of the lower channel. The wave function of the bound state of vanishing energy in channel 2 has, indeed, little overlap with the configuration of channel 1. In other words, $\alpha_{1}$ and $\beta_{1}$ in Equations (1) and (2) would be close to zero. Hence, in this region, the dynamics are dominated by the attraction in the upper channel and the second channel is mainly a tool for detection.

The results shown in Figure 5 fit nicely with the recent observations by the $\mathrm{LHCb}$ Collaboration. We have compiled in Table 3 the most common suggestions of molecular structure for the $P_{c}^{+}$pentaquarks (note that the $\Lambda_{c}^{+} \bar{D}^{0}$ interactions are expected to be repulsive, leaving only $\Sigma_{c}^{+} \bar{D}^{0}$ pairs, which are expected to form bound states [24]), all of them detected in the $J / \psi p$ channel. Let us first concentrate on the most recent results from the LHCb Collaboration [3], quoted in the last three lines of Table 3. The phase space for the decay to $J / \psi p$ has been given in Table 1, the resonances being far from the detection channel. When this happens, we have seen in Figure 5 that it is the mass difference in comparison to the formation channel that determines the width of the resonance; the larger the binding the larger the width. The binding energies of the recent $P_{c}^{+}$pentaquarks are, in increasing order (see Table 3): $2.5 \mathrm{MeV}, 5.8 \mathrm{MeV}$ and $19.5 \mathrm{MeV}$, and the corresponding decay widths are (see Table 1): $6.4 \mathrm{MeV}, 9.8 \mathrm{MeV}$ and $20.6 \mathrm{MeV}$, in perfect agreement with our results.

The validity of our arguments has a relevant side consequence about the nature of the $P_{c}^{+}(4380)$ pentaquark, whose structure is awaiting an amplitude analysis of additional data [25], to prove that this state is not an artifact of interfering with $\Lambda^{*}$ resonances [3]. This broad structure cannot have its origin in the attractive interaction of the $\Sigma_{c}^{*+} \bar{D}^{0}$ channel; that has been suggested mainly due to its vicinity to the mass of the resonance. We then 
conclude that the formation channel responsible for the $P_{c}^{+}(4380)$ pentaquark should be at a similar energy distance as the detection channel to maximize its width according to Figure 5. If the $P_{c}^{+}(4380)$ does turn out to be a genuine resonance after all, it is very unlikely that its nature would be correlated with a $\Sigma_{c}^{*} \bar{D}$ molecular state [26]. The true nature of the $P_{c}^{+}(4380)$ is still an intriguing issue, and is an outstanding challenge for future experiments [27].

Table 3. Suggested molecular structures of the $P_{c}^{+}$pentaquarks $[2,3,23,25]$.

\begin{tabular}{cccc}
\hline State & Suggested Molecule & $\boldsymbol{M}_{\text {molecule }}(\mathbf{M e V})$ & $\boldsymbol{\Delta E} \mathbf{( M e V )}$ \\
\hline$P_{c}(4380)^{+}$ & $\Sigma_{c}^{*+} \bar{D}^{0}$ & $4382.3 \pm 2.3$ & $2.3 \pm 30.1$ \\
$P_{c}(4312)^{+}$ & $\Sigma_{c}^{+} \bar{D}^{0}$ & $4317.7 \pm 0.4$ & $5.8 \pm 6.8$ \\
$P_{c}(4440)^{+}$ & $\Sigma_{c}^{+} \bar{D}^{*^{0}}$ & $4459.8 \pm 0.4$ & $19.5 \pm 4.9$ \\
$P_{c}(4457)^{+}$ & $\Sigma_{c}^{+} \bar{D}^{*^{0}}$ & $4459.8 \pm 0.4$ & $2.5 \pm 4.1$ \\
\hline
\end{tabular}

\subsection{Dependence of the Decay Width on $\Delta M$}

We now wonder if the results obtained in the previous subsection critically depend on the relative distance between the coupled channels. To answer this question, we have generated a second scenario with a different value of $\Delta M$. In this case we have reduced the value of $\Delta M$ by a factor five. We start with the same relative position of the resonance with respect to $\Delta M$, corresponding to the purple circle in Figure 6 . The results are shown by the dashed line in Figure 6. As can be seen, the behavior of the decay width is rather similar to that obtained in Section 3.2. Although it was to be expected, this result has a rather important consequence, as it is the rapid variation of the decay width of a resonance generated in between coupled-channels that are very close-a small value of $\Delta M$. In this case, the decay width of a resonance changes by more than a factor of three in a small interval of around $5 \mathrm{MeV}$. Thus, for resonances that are generated in between rather close channels, the position of the resonance is crucial for the knowledge of its decay width. For larger values of $\Delta M$, the variation of the decay width proceeds more smoothly.

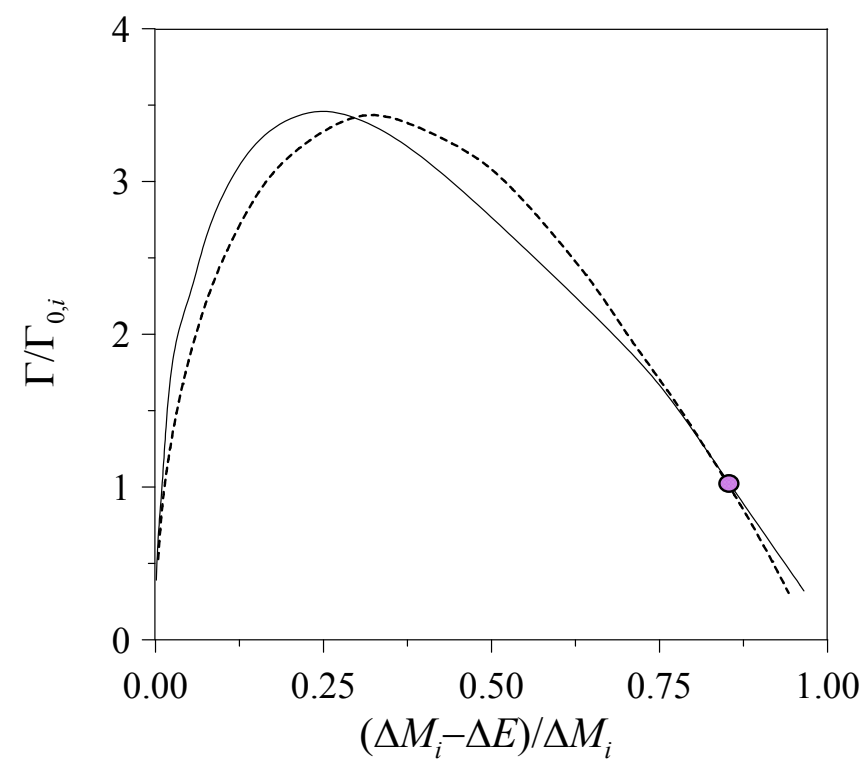

Figure 6. Variation of the decay width of a resonance, $\Gamma$ (in units of $\Gamma_{0, i}$ ), as a function of the relative position of the resonance in between the formation and the detection channels $\Delta M_{i}-\Delta E$ (in units of $\Delta M_{i}$ ) for two different values of $\Delta M_{i}: \Delta M_{1}=\Delta M_{0}$, solid line, and $\Delta M_{2}=\Delta M_{0} / 5$, dashed line. The width of the resonance for the starting point in each case is denoted by $\Gamma_{0, i}$. See text for details

This scenario applies in a symmetric manner to one of the most elusive exotic states in the meson sector, which, however, has been firmly established by different collabora- 
tions and the properties of which seem to be hardly accommodated in a quark-antiquark scheme - this is the $X(3872)$ [28] (note, however, that there exist studies [29] that find a good fit of the data with mainly a $c \bar{c}$ structure). Its small width, $\Gamma<1.2 \mathrm{MeV}$, would fit our argumentation if considered as a coupled-channel resonance of $D^{0} \bar{D}^{* 0}-D^{+} \bar{D}^{*-}$, whose mass difference is $7-8 \mathrm{MeV}$ and which is rather close to the lowest channel $[13,30]$. There could also be such a contribution from other components, such as $J / \psi \omega$ [30], that it is almost degenerate with the upper channel $D^{+} \bar{D}^{*-}$, and our conclusions would remain.

\subsection{Dependence of the Decay Width on $\Delta E$}

We do finally present the study of the variation of the decay width of a resonance increasing the binding energy $\Delta E$ for a fixed value of its mass with respect to the detection channel $\Delta M-\Delta E$. For this purpose, we have diminished the mass of the detection channel combined with an increase of the attractive term, $A^{11}$, in the interaction of the detection channel. The results are presented in Figure 7. As could have been expected on the basis of the results shown in Figure 5, the larger the binding energy, $\Delta E$, the larger the decay width, $\Gamma$, of the resonance, despite having a fixed phase space. This result shows neatly how the width provides basic information about the coupled channels that contribute to the formation of a resonance.

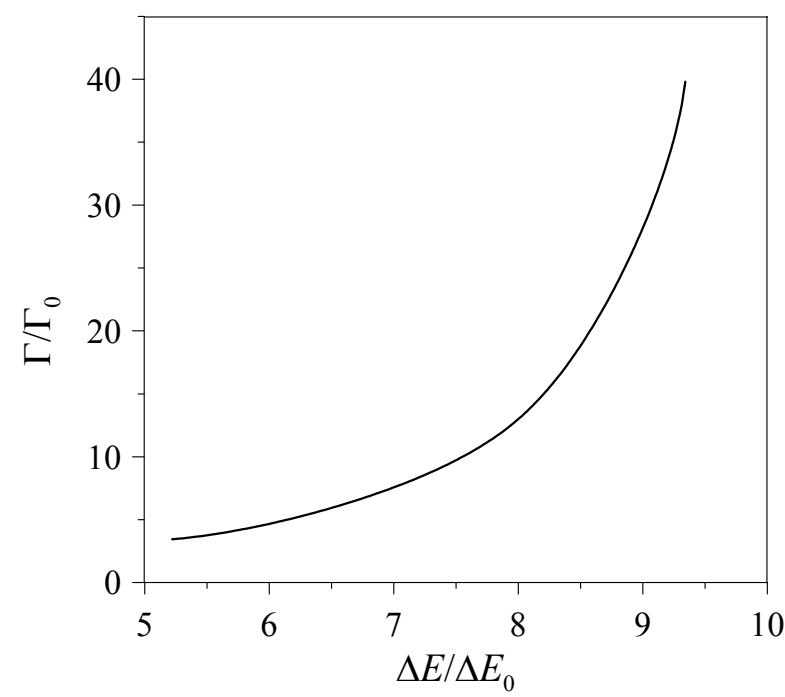

Figure 7. Variation of the decay width of a resonance, $\Gamma$ (in units of $\Gamma_{0}$ ), as a function of the binding energy, $\Delta E$ (in units of $\Delta E_{0}$ ) for a fixed phase space, $\Delta M-\Delta E$.

Let us make a final comment regarding the possible existence of partners of multiquark states in different flavor sectors. Based on the results presented above, the dynamics of a coupled channel multiquark state made up of different color singlet components are strongly constrained by the relative position of the internal thresholds. As has been illustrated in Figures 1 and 2, the relative position of the thresholds varies a lot depending on the flavor sector, symmetrically, for $B=0$ and $B=1$. As a consequence, if a resonance is generated by the coupling between the internal thresholds, moving from the charm to the bottom sector would have an important effect to be considered-the augment of the mass difference between the thresholds. This would translate into a less effective channel coupling, the non-diagonal matrix elements of a flavor-independent dominated potential being divided by the mass difference between the coupled channels. On the other hand, if the resonance is a consequence of the attraction in the formation channel, the lower channel being just a tool for detection, then the resulting state in the heavier flavor sector could have very different properties due to the relative position of the formation and detection channels with respect to the resonance. In this case, our formalism provides an excellent tool for guessing the existence, and predicting the properties, of possible partners in different flavor sectors. 


\section{Outlook}

We have presented a comprehensive study of the decay width of multiquark states made up of different color singlet components. We show how the decay width can provide in-depth information about the nature of a coupled-channel resonance. The symmetrical situation between meson- and baryon-like multiquarks has been highlighted. One may conclude that an unexpected behavior of the decay width of a multiquark state could be pointing to a relevant role of the coupled-channel dynamics, aiming at the channel responsible for the formation of the resonance.

The scenario we have presented accounts for the existence of narrow resonances with large phase spaces. In the case of resonances that are far from the detection channel, it is the mass difference with respect to the formation channel that determines the decay width of the resonance. The larger the binding, the larger the decay width, despite a reduced phase space. This is clearly the case in the recently reported LHCb pentaquarks: $P_{c}(4312)^{+}$, $P_{c}(4440)^{+}$and $P_{c}(4457)^{+}$. Our results cast doubts on the $\Sigma_{c}^{*} \bar{D}$ molecular nature of the $P_{c}^{+}(4380)$ pentaquark. According to our results, if the $P_{c}^{+}(4380)$ does turn out to be a genuine resonance after all, the channel responsible for the formation molecular structure should be at a similar energy distance as the detection channel in order to maximize its width. Another relevant outcome of our study is the rapid variation of the decay width of a coupled-channel resonance generated in between thresholds with a very small mass difference. In this case, the decay width of a resonance could change by more than a factor of three in a small interval of a few MeV. This would apply, among others, to the X(3872).

Let us finally note that, although the exact shape of the dependence of the decay width of a coupled-channel resonance on its position with respect to the detection channel would rely on the specific dynamics of the coupled-channel system, the gross features obtained in this study might be a relevant and basic hint for exploring the nature of multiquark states. Our analysis also provides clues to the existence and properties of the partners of already known resonances in different flavor sectors. A certain caution is needed when predicting partners and/or the properties of known multiquarks in different flavor sectors if the dynamics are governed by coupled channels.

Author Contributions: Conceptualization, H.G. and A.V.; Methodology, H.G. and A.V.; Formal analysis, H.G. and A.V.; Investigation, H.G. and A.V.; Writing-original draft preparation, H.G. and A.V.; Writing-review and editing, H.G. and A.V. All authors have read and agreed to the published version of the manuscript.

Funding: This research was funded by COFAA-IPN (México) and by Ministerio de Economía, Industria y Competitividad and EU FEDER under Contracts No. PID2019-105439GB-C22 and RED2018102572-T.

Institutional Review Board Statement: Not applicable.

Informed Consent Statement: Not applicable.

Data Availability Statement: Not applicable.

Conflicts of Interest: The authors declare no conflict of interest.

\section{References}

1. Liu, Y.-R.; Chen, H.-X.; Chen, W.; Liu, X.; Zhu, S.-L. Pentaquark and Tetraquark States. Prog. Part. Nucl. Phys. 2019, 107, 237-320. [CrossRef]

2. Aaij, R.; Adeva, B.; Adinolfi, M.; Affolder, A.; Ajaltouni, Z.; Akar, S.; Albrecht, J.; Alessio, F.; Alexander, M.; Ali, S.; et al. (LHCb Collaboration). Observation of $J / \psi p$ Resonances Consistent with Pentaquark States in $\Lambda_{b}^{0} \rightarrow J / \psi K^{-} p$ Decays. Phys. Rev. Lett. 2015, 115, 072001. [CrossRef]

3. Aaij, R.; Abellán Beteta, C.; Adeva, B.; Adinolfi, M.; Aidala, C.A.; Ajaltouni, Z.; Akar, S.; Albicocco, P.; Albrecht, J.; Alessio, F.; et al. (LHCb Collaboration). Observation of a Narrow Pentaquark State, $P_{c}(4312)^{+}$, and of the Two-Peak Structure of the $P_{c}(4450)^{+}$ Phys. Rev. Lett. 2019, 122, 222001. [CrossRef]

4. Brau, F.; Semay, C.; Silvestre-Brac, B. Unified meson-baryon potential. Phys. Rev. C 2002, 66, 055202. [CrossRef] 
5. Isgur, N. Beyond the adiabatic approximation: The impact of thresholds on the hadronic spectrum. Phys. Rev. D 1999, 60, 054013. [CrossRef]

6. Ablikim, M.; Achasov, M.N.; Adlarson, P.; Ahmed, S.; Albrecht, M.; Aliberti, R.; Amoroso, A.; An, Q.; Anita; Bai, X.H.; et al. (BESIII Collaboration). Observation of a Near-Threshold Structure in the $K^{+}$Recoil-Mass Spectra in $e^{+} e^{-} \rightarrow K^{+}\left(D_{s}^{-} D_{0}^{*}+D_{s}^{*-} D^{0}\right)$. Phys. Rev. Lett. 2021, 126, 102001. [CrossRef]

7. Fredriksson, S.; Jande, M. Diquark Deuteron. Phys. Rev. Lett. 1982, 48, 14-16. [CrossRef]

8. Vijande, J.; Valcarce, A. Probabilities in nonorthogonal bases: Four-quark systems. Phys. Rev. C 2009, 80, 035204. [CrossRef]

9. Richard, J.-M.; Valcarce, A.; Vijande, J. Stable heavy pentaquarks in constituent models. Phys. Lett. B 2017, 774, 710-714. [CrossRef]

10. Close, F. The End of the Constituent Quark Model? AIP Conf. Proc. 2004, 717, 919-936.

11. Weinstein, J.D.; Isgur, N. KK molecules. Phys. Rev. D 1990, 41, 2236-2257. [CrossRef]

12. Zyla, P.A.; Barnett, R.M.; Beringer, J.; Bonventre, R.J.; Dahl, O.; Dwyer, D.A.; Groom, D.E.; Lin, C.-J.; Lugovsky, K.S.; Pianori, E.; et al. (Particle Data Group). Review of Particle Physics. Prog. Theor. Exp. Phys. 2020, 2020, 083 C01.

13. Braaten, E.; Kusunoki, M. Low-energy universality and the new charmonium resonance at 3870 MeV. Phys. Rev. D 2004 69, 074005. [CrossRef]

14. Pilloni, A. Exotic Hadrons as Feshbach Resonances. Act. Phys. Pol. B Proc. Supp. 2014, 7, 463. [CrossRef]

15. Caramés, T.F.; Valcarce, A.; Vijande, J. Too many X's, Y's and Z's? Phys. Lett. B 2012, 709, 358-361. [CrossRef]

16. Malfliet, R.A.; Tjon, J.A. Solution of the Faddeev equations for the triton problem using local two particle interactions. Nucl. Phys. A 1969 127, 161-168. [CrossRef]

17. Filikhin, I.; Suslov, V.M.; Vlahovic, B. Faddeev calculations for light $\Xi^{-}$hypernuclei. Math. Model. Geom. $2017,5,1-11$.

18. Breit, G.; Wigner, E. Capture of Slow Neutrons. Phys. Rev. 1936, 49, 519-531. [CrossRef]

19. Ceci, S.; Švarc, A.; Zauner, B.; Manley, D.M.; Capstick, S. Model-independent resonance parameter extraction using the trace of K and T matrices. Phys. Lett. B 2008, 659, 228-233. [CrossRef]

20. Ceci, S.; Korolija, M.; Zauner, B. Model-Independent Extraction of the Pole and Breit-Wigner Resonance Parameters. Phys. Rev. Lett. 2013, 111, 112004. [CrossRef]

21. Garcilazo, H.; Valcarce, A. Effect of thresholds on the width of three-body resonances. Phys. Lett. B 2017, 772, 394-397. [CrossRef]

22. Garcilazo, H.; Valcarce, A. Width of a two-body coupled-channel resonance. Eur. Phys. J. C 2018, 78, 259. [CrossRef]

23. Burns, T.J. Phenomenology of $P_{c}(4380)^{+}, P_{c}(4450)^{+}$and related states. Eur. Phy. J. A 2015, 51, 152. [CrossRef]

24. Wu, J.-J.; Lee, T.-S.H.; Zou, B.S. Nucleon resonances with hidden charm in coupled-channels models. Phys. Rev. C 2012, 85, 044002. [CrossRef]

25. Karliner, M.; Rosner, J.L. Strange pentaquarks and excited $\Xi$ hyperons in $\Xi_{b} \rightarrow J / \psi \Lambda K^{-}$final states. To appear in Science Bulletin. arXiv 2021, arXiv:2104.15077.

26. Chen, R.; Liu, X.; Li, X.-Q.; Zhu, S.-L. Identifying Exotic Hidden-Charm Pentaquarks. Phys. Rev. Lett. 2015, 115, 132002. [CrossRef]

27. Karliner, M.; Rosner, J.L. New Exotic Meson and Baryon Resonances from Doubly Heavy Hadronic Molecules. Phys. Rev. Lett. 2015, 115, 122001. [CrossRef]

28. Choi, S.K.; Olsen, S.L.; Abe, K.; Abe, T.; Adachi, I.; Ahn, B.S.; Aihara, H.; Akai, K.; Akatsu, M.; Akemoto, M.; et al. (Belle Collaboration). Observation of a Narrow Charmoniumlike State in Exclusive $B^{ \pm} \rightarrow K^{ \pm} \pi^{+} \pi^{-} J / \psi$ Decays. Phys. Rev. Lett. 2003, 91, 262001. [CrossRef]

29. Meng, C.; Sanz-Cillero, J.J.; Shi, M.; Yao, D.-L.; Zheng, H.-Q. Refined analysis on the X(3872) resonance. Phys. Rev. D 2015, 92, 034020. [CrossRef]

30. Braaten, E.; Lu, M. Effects of charged charm mesons on the line shapes of the X(3872). Phys. Rev. D 2008, 77, 014029. [CrossRef] 